\title{
RAMSEY THEOREMS FOR MULTIPLE COPIES OF GRAPHS
}

\author{
BY
}

\author{
S. A. BURR, P. ERDÖS AND J. H. SPENCER( $\left.{ }^{1}\right)$
}

\begin{abstract}
If $G$ and $H$ are graphs, define the Ramsey number $r(G, H)$ to be the least number $p$ such that if the edges of the complete graph $K_{p}$ are colored red and blue (say), either the red graph contains $G$ as a subgraph or the blue graph contains $H$. Let $m G$ denote the union of $m$ disjoint copies of $G$. The following result is proved: Let $G$ and $H$ have $k$ and $l$ points respectively and have point independence numbers of $i$ and $j$ respectively. Then $N-1 \leqslant$ $r(m G, n H) \leqslant N+C$, where $N=k m+l n-\min (m i, m j)$ and where $C$ is an effectively computable function of $G$ and $H$. The method used permits exact evaluation of $r(m G, n H)$ for various choices of $G$ and $H$, especially when $m=n$ or $G=H$. In particular, $r\left(m K_{3}, n K_{3}\right)=3 m+2 n$ when $m \geqslant n, m \geqslant 2$.
\end{abstract}

1. Introduction. Let $G$ and $H$ be graphs without isolated points. Following Chvátal and Harary [1], define the Ramsey number $r(G, H)$ to be the least integer $n$ such that if the edges of $K_{n}$ (the complete graph on $n$ pcints) are two-colored, say red and blue, either the red graph contains $G$ as a subgraph or the blue graph contains $H$. Note that $r\left(K_{k}, K_{l}\right)$ is the "ordinary" Ramsey number $r(k, l)$ for which an extensive literature exists. The evaluation of $r(G, H)$ has received attention from several authors in recent years. An extensive survey is given in [2].

In this paper we will generally follow the notation of Harary [3]. In particular, let $n G$ denote the union of $n$ vertex-disjoint copies of $G$. In $\S 2$ we obtain surprisingly sharp and general upper and lower bounds for $r(n G, n H)$ for $G$ and $H$ fixed and $n$ sufficiently large. In $\S 3$ we extend these results to $r(m G, n H)$, in $\S 4$ to $k$-graphs. In $\S 5$ we consider a related problem of J. W. Moon concerning the decomposition of a complete graph into complete monochromatic subgraphs of prescribed size. Finally, in $\S 6$ we give exaet values for various cases.

2. The Ramsey numbers $r(n G, n H)$. Again following [3], let $p(G)$ denote the number of points of $G$ and let $\beta_{0}(G)$ denote the number of points in a maximal independent set in $G$. As a special notation, let $[X]^{2}$ denote the complete graph on $X$ and $X Y$ denote the complete bipartite graph on $X$ and $Y$. Also, let $r(G)=r(G, G)$; these we call the diagonal Ramsey numbers.

Presented to the Society, January 16, 1974; received by the editors January 14, 1974. AMS (MOS) subject classifications (1970). Primary 05C35; Secondary 05C15.

(1) Supported under U.S. Office of Naval Research Contract N00014-67-A-0202-0063. 
THEOREM 1. Let $p(G)=k, p(H)=l$, and $i=\min \left(\beta_{0}(G), \beta_{0}(H)\right)$. Then

$$
(k+l-i) n-1 \leqslant r(n G, n H) \leqslant(k+l-i) n+C,
$$

where $C$ is a constant depending only on $G$ and $H$.

We first prove Theorem 1 for $G=H=K_{3}$. The more general proof to follow will then have clearer intuitive appeal. In fact, we show the following stronger result, which has been shown independently by Seymour at Oxford (personal communication).

THEOREM 2. For $n \geqslant 2, r\left(n K_{3}\right)=5 n$.

Before turning to the proof of Theorem 2, we prove the following simple result which will be used several times in the sequel.

Lemma 1. Let $F, G$, and $H$ be graphs, with $p(G)=k, p(H)=l$. Then, if $m, n \geqslant 1$,

$$
\begin{aligned}
& r(G, F \cup H) \leqslant \max (r(G, F)+l, r(G, H)), \\
& r(m G, n H) \leqslant r(G, H)+(m-1) k+(n-1) l .
\end{aligned}
$$

Proof. Let a complete graph on $\max (r(G, F)+l, r(G, H))$ points be twocolored. If there is no red $G$, then there is certainly a blue $H$. Remove the $l$ points of $H$ from the graph. Among the remaining points there must be a blue $F$. Hence the original graph contains either a red $G$ or a blue $F \cup H$, and the first inequality follows. The second inequality follows from repeated application of the first. Q.E.D.

Proof of Theorem 2. We show $r\left(n K_{3}\right) \geqslant 5 n$ by exhibiting the coloring of Figure 1:

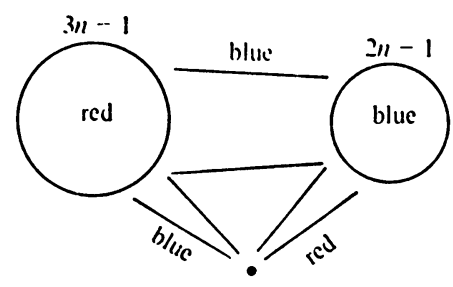

FiguRE 1

Formally, let $|A|=3 n-1,|B|=2 n-1,|C|=1$, with $A, B, C$ disjoint. Color $[A \cup B \cup C]^{2}$ by coloring $[A]^{2}$ red, $[B]^{2}$ blue, $A B$ blue, $A C$ blue, and $B C$ red. The reader can easily show that this is a two-coloring of $K_{5 n-1}$ without a monochromatic $n K_{3}$.

We show $r\left(n K_{3}\right) \leqslant 5 n$ by induction. The finite demonstration for $n=2$ is 
given in $\S 6$. Now let $n \geqslant 3$, and fix a two-coloring of $K_{5 n}$. We need to show the existence of a monochromatic $n K_{3}$. Since $5 n \geqslant 6$, there exists a monochromatic, say red, $K_{3}$.

Assume there is no blue $K_{3}$ on the remaining $5 n-3$ points. Then, since $r\left(K_{3}\right)=6$, by Lemma 1 we have $r\left(n K_{3}, K_{3}\right) \leqslant 3 n+3 \leqslant 5 n-3$, because $n \geqslant 3$. Since there is no blue $K_{3}$, there must be $n$ disjoint red $K_{3}$ 's as desired.

Now assume, on the other hand, that there is a blue $K_{3}$ which is vertexdisjoint from the red $K_{3}$. That is $|A|=|B|=3, A \cap B=\varnothing,[A]^{2}$ red, $[B]^{2}$ blue. (See Figure 2(a).)

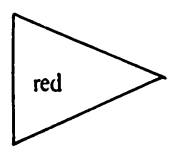

(a)

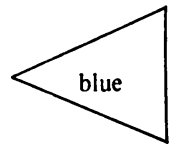

\section{Figure 2}

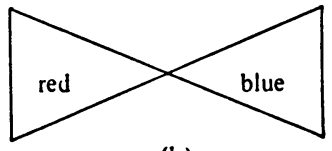

(b)

Of the nine lines of $A B$ at least five must be one color, say red. Then $\exists a \in A$ and $b_{1}, b_{2} \in B$, such that $\left\{a, b_{1}\right\},\left\{a, b_{2}\right\}$ are red. This yields (see Figure 2(b)) a "bowtie": two $K_{3}$, one red, one blue, with one common vertex. Deleting the bowtie we find, by the induction hypothesis, a monochromatic $(n-1) K_{3}$. Adding the appropriately colored $K_{3}$ from the bowtie yields a monochromatic $n K_{3}$ in the full graph. Q.E.D. (Theorem 2).

The next result, which is essentially the lower bound in Theorem 1, provides a very useful lower bound for Ramsey numbers in general and is therefore given separately.

LEMMA 2. If $p(G)=k, p(H)=l$, then

$$
r(G, H) \geqslant k+l-\min \left(\beta_{0}(G), \beta_{0}(H)\right)-1 .
$$

Proof. We form a graph on $k+l-\beta_{0}(G)-2$ points containing neither a red $G$ nor a blue $H$. Let $|A|=k-\beta_{0}(G)-1,|B|=l-1, A \cap B=\varnothing$, and color $[B]^{2}$ blue, all else red (see Figure 3).

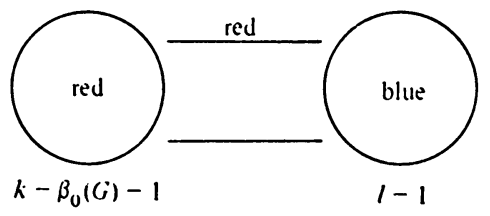

FIGURE 3

It is clear that there is no blue $H$; but a red $G$ would have to use $\beta_{0}(G)+1$ points of $B$, which is impossible since they would all be independent in the red graph. Thus $r(G, H) \geqslant k+l-\beta_{0}(G)-1$; but by symmetry $r(G, H) \geqslant k+l-\beta_{0}(H)-1$, 
and combining the two inequalities yields the desired result. Q.E.D.

Proof of THEOREM 1. Without loss of generality, we may assume $\beta_{0}(G) \geqslant$ $\beta_{0}(H)=i$. For the lower bound of (1) employ Lemma 2, with $G$ replaced by $n G$ and $H$ replaced by $n H$. Then $k$ and $l$ become $n k$ and $n l$. Thus, since $\beta_{0}(n G)=$ $n \beta_{0}(G)$ and $\beta_{0}(n H)=n \beta_{0}(H)$, we have

$$
\begin{aligned}
r(n G, n H) & \geqslant n k+n l-\min \left(\beta_{0}(n G), \beta_{0}(n H)\right)-1 \\
& =n(k+l-i)-1 .
\end{aligned}
$$

We now show the upper bound in (1). Let $n_{0}$ be a constant, dependent only on $G$ and $H$, to be described later. We find $C \geqslant 0$ so that (1) holds for $n \leqslant n_{0}$, and now wish to apply induction. Fix a two-coloring $\chi$ of the edges of the complete graph on $(k+l-i)(n+1)+C$ points; we need to show the existence of a red $(n+1) G$ or a blue $(n+1) H$. Here $n \geqslant n_{0}$, and we know $r(n G, n H) \leqslant$ $(k+l-i) n+C$.

By a "bowtie" we mean in this case a two-colored graph on $\leqslant k+l-i$ points containing a red $G$ and a blue $H$. An example of such a graph is one on the set of points $S=R \cup N \cup B$ where $|R|=k-i,|B|=l-i,|N|=i$, all disjoint, $[R]^{2}$ red, $[B]^{2}$ blue, $R N$ red, $B N$ blue, and the other edges unspecified. (See Figure 4.)

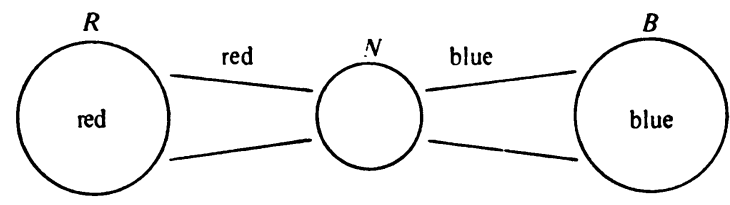

Figure 4. Bowtie

If the graph contains a bowtie then, deleting the bowtie, we find by induction a monochromatic $n G$ or $n H$, to which we add the appropriately colored $G$ or $H$ from the bowtie giving a red $(n+1) G$ or a blue $(n+1) H$.

Let $M \geqslant \max (k, l)-i$ be such that if $|A|=|D|=M, A \cap D=\varnothing$, and $A D$ is two-colored, there exist $A_{1} \subseteq A, D_{1} \subseteq D$ with $\left|A_{1}\right|=\left|D_{1}\right|=\max (k, l)-i$ and $A_{1} D_{1}$ monochromatic. (The existence of such an $M$ is not hard to verify; one may take $M=2^{\max (k, l)+1}$ for definiteness.) Assume, given a coloring $\chi$, that there exist $A, D$ with $|A|=|D|=M, A \cap D=\varnothing,[A]^{2}$ red, $[D]^{2}$ blue. We find $A_{1}, D_{1}$, with $A_{1} D_{1}$ monochromatic. (See Figure 5.)

If $A_{1} D_{1}$ is red, we torm a bowtie by taking $R$ to be $k-i$ points out of $A_{1}$, $N$ to be $i$ points out of $D_{1}$, and $B$ to be $l-i$ other points of $D$. If $A_{1} D_{1}$ is blue, we proceed similarly, taking $N$ out of $A_{1}$.

Finally, assume there do not exist monochromatic vertex-disjoint $K_{M}$ of 


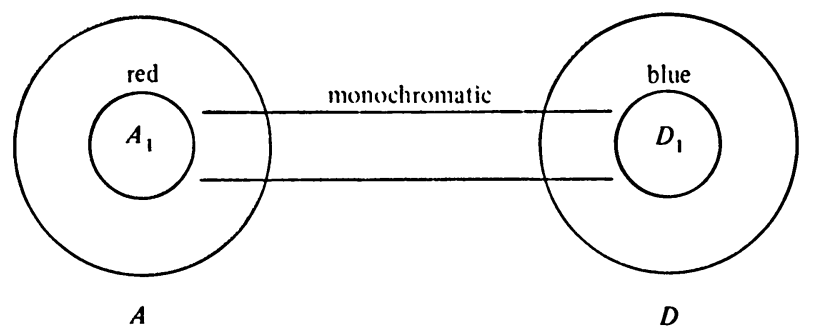

FIGURE 5. Large red and blue $K_{M}$ yielding bowtie different colors. For convenience, let $y=(n+1)(k+l-i)+C=$ number of points. If $y \geqslant r(M, M)$ we find a monochromatic $K_{M}$, say red. By Lemma 1 ,

$$
r\left((n+1) G, K_{M}\right) \leqslant r\left(G, K_{M}\right)+n k \leqslant r(k, M)+n k .
$$

Thus, provided $y \geqslant r(M, M)$ and $y-M \geqslant r(k, M)+n k$, we either have a red $(n+1) G$, in which case we are done, or else we have a blue $K_{M}$ disjoint from the red one, a contradiction. Now we specify $n_{0}$ to be an integer such that

$$
\begin{aligned}
\left(n_{0}+1\right)(k+l-i) & \geqslant r(M, M), \\
\left(n_{0}+1\right)(k+l-i)-M-r(k, M) / p & \geqslant n_{0} k_{0}
\end{aligned}
$$

Note that the definition of $n_{0}$ depends only on $G$ and $H$-in fact, only on $k$ and l. (Since $C$ does not appear in the definition of $n_{0}$, we have avoided circularity.) To summarize, given $n \geqslant n_{0}$ and a coloring $\chi$ on the complete graph on $(k+l-i)(n+1)+C$ points, one of the following holds:

(a) We find two monochromatic $K_{M}$ of different colors, in which case there exists a bowtie so, by induction, a monochromatic $(n+1) G$; or

(b) there is no $K_{M}$ in one color, in which case the use of Lemma 1 gives an $(n+1) G$ in the other color. Q.E.D. (Theorem 1).

Note that in our proof of Theorem 1 we actually showed

(1) $(k+l-i) n-1 \leqslant r(n G, n H)$,

(2) $r((n+1) G,(n+1) H) \leqslant r(n G, n H)+(k+l-i)$ for $n \geqslant n_{0}$.

The function $g(n)=r(n G, n H)-(k+l-i) n$ is integral, nonincreasing for $n \geqslant n_{0}$, and bounded from below. Consequently, it is eventually constant. Hence, the following theorem.

THEOREM 3. Under the assumptions of Theorem 1, there exist $n_{1}$ and $C_{1}$ such that

$$
r(n G, n H)=(k+l-i) n+C_{1} \text { for } n \geqslant n_{1} .
$$

We note that we have not been able to find any upper bound on $n_{1}$. That is, we have not been able to show that $n_{1}$ is a recursive function of $G$. 
3. The Ramsey numbers $r(m G, n H)$.

TheOrem 4. Let $p(G)=k, p(H)=l, \beta_{0}(G)=i$, and $\beta_{0}(H)=j$. Then

$$
\begin{aligned}
k m+l n-\min (m i, n j)-1 & \leqslant r(m G, n H) \\
& \leqslant k m+\ln -\min (m i, n j)+C,
\end{aligned}
$$

where $C$ is a constant depending only on $G$ and $H$.

Proof. The lower bound follows directly from Lemma 2. We will prove the upper bound by induction on $m+n$. Starting the induction is trivial (for a suitable value of $C$ ). Now assume the result to have been proved for all cases in which $m+n$ is less than some value $N$, and consider a case in which $m+n=N$. If either $m$ or $n$ is no greater than $\max (i, j)$, then by Lemma 1 the desired inequality holds, with some new value for $C$.

By a bowtie we will now mean a two-colored graph on $k j+l i-i j$ joints containing simultaneously a red $j G$ and a blue $i H$. Suppose now we have a twocoloring on $\mathrm{km}+\ln -\min (m i, n j)+C$ points which contains a bowtie. On removing the bowtie one has a graph on

$$
\begin{aligned}
k m+l n-\min (m i, n j)+ & C-k j-l i+i j \\
& =k(m-j)+l(n-i)-\min ((m-j) i,(m-i) j)+C
\end{aligned}
$$

points. By the induction hypothesis, this graph contains either a red $(m-j) G$ or a blue $(n-i) H$, and hence the original graph contains either a red $m G$ or a blue $n H$. The argument is almost the same as that in the proof of Theorem 1 and will only be sketched. Take $M=2^{\max (k j, l i)+1}$. Then, if $C$ has been chosen large enough, the graph contains a monochromatic (say red) $K_{M}$. By Lemma 1 , and again assuming $C$ is large enough, the rest of the graph contains either a red $m G$ or a blue $K_{M}$. In the former case we are done immediately; in the latter case we have a monochromatic complete bipartite graph joining the two $K_{M}$ sufficiently large to guarantee the existence of a bowtie. Q.E.D.

It is possible to prove a considerably more general result than Theorem 4, which may be stated as follows: Let $G$ and $H$ be disjoint unions of graphs chosen from a finite set $G$ of graphs, and let $p(G)=k, p(H)=l, \beta_{0}(G)=i$, and $\beta_{0}(H)=j$. Then $k+l-\min (i, j)-1 \leqslant r(G, H) \leqslant k+l-\min (i, j)+C$, where $C$ depends only on $G$. Although the ideas involved in the proof are essentially the same as those in this paper, the details are tedious and will be omitted.

4. $k$-graphs. In this section we partially extend the results of Theorem 1 to $k$-graphs. A $k$-graph is defined as a set $V$ of vertices and a set $E$ of "edges" where each edge $e \in E$ is a subset of $V$ of cardinality $k$. It is clear that general- 
ized Ramsey theory can be extended to $k$-graphs, and indeed has been discussed in [4]. Our proofs will be more sketchy than in the previous sections; also, for clarity's sake, only diagonal numbers will be considered.

THEOREM 5. Let $G$ be a $k$-graph with no isolated points. Then

$$
D n-1 \leqslant r(n G) \leqslant D n+C,
$$

where $D=D(G)$ will be defined in the proof and $C$ is a constant depending only on $G$.

Proof. Let $G$ have $p$ points. Let $[X]^{k}=\{Y \subseteq X:|Y|=k\}$ denote the complete $k$-graph on $X$. Let $A, B$ be disjoint sets. A coloring $c$ of $[A \cup B]^{k}$ is called canonical if the color of $e \in[A \cup B]^{k}$ is dependent only on $|e \cap A|$. There are only $2^{k+1}$ canonical colorings, corresponding to $(k+1)$-tuples of red and blue. Let $c$ be such a coloring where $|A|,|B|$ are sufficiently large, with $[A]^{k}$ red, $[B]^{k}$ blue. Let $r_{c}$ be the least integer such that $r_{c}$ points from $A$ and $p-r_{c}$ points from $B$ contain a red $G\left(r_{c} \leqslant p\right.$, since $[A]^{k}$ is red). Let $b_{c}$ be the largest integer such that $b_{c}$ points from $A$ and $p-b_{c}$ points from $B$ contain a blue $G$ (note that $b_{c} \geqslant 0$ ). Let

$$
D_{c}= \begin{cases}p+r_{c}-b_{c} & \text { if } r_{c} \geqslant b_{c}, \\ p & \text { if } r_{c} \leqslant b_{c} .\end{cases}
$$

If $D_{c}>p$ then $r_{c}$ points from $A$ together with $p-b_{c}$ points from $B$ contain both a red and a blue $G$. That is, there is a bowtie on $D_{c}$ points. If $D_{c}=p$, so $r_{c} \leqslant b_{c}$, then $r_{c} b_{c}$ points from $A$ together with $\left(p-r_{c}\right) b_{c}$ points from $B$ form both a red $b_{c} G$ (each $G$ split $\left.r_{c}, p-r_{c}\right)$ and a blue $b_{c} G\left(r_{c} G\right.$ 's split $b_{c}, p-b_{c}$; the other $\left(b_{c}-r_{c}\right) G$ 's entirely in $\left.B\right)$. That is, there are $p b_{c}$ points that contain a red and a blue $b_{c} G$. We shall call this a multibowtie.

We define $D=\min D_{c}$ over the $2^{k-1}$ possible $c$ with $[A]^{k}$ red and $[B]^{k}$ blue. The lower bound of Theorem 5 is trivial if $D=p$. If not, let $c$ be such that $D=D_{c}$. Split $n D-2$ points into $A, B,|A|=n r_{c}-1,|B|=n b_{c}-1$, and color $[A \cup B]^{k}$ canonically by $c$.

The upper bound is by induction, beginning with $n$ sufficiently large. Fix a coloring $c$. Then the $k$-graph so colored must contain disjoint sets $X, Y$, colored red and blue, where $|X|,|Y|$ are arbitrarily large (though independent of $n$ ); for otherwise, by analogy with Lemma 1 the points may be split into $G$ 's of the same color with a bounded number of points left over-so $r(n G)$ would be $\leqslant n p+C$. We pick $|X|,|Y|$ so large that there must be $A \subseteq X, B \subseteq Y$ so that $A \cup B$ is colored canonically. (This step requires strong use of Ramsey's theorem and yields absurdly high bounds.) Say $A \cup B$ is colored canonically by $c$; of course $D_{c} \geqslant D$. We find a bowtie of $D$ points (or perhaps, if $D_{c}=p$, a multi- 
bowtie). Deleting it and applying induction, we arrive at a proof of Theorem 5 . Q.E.D.

We note that the off-diagonal number could also be easily found.

Corollary. Let $K_{p}^{(k)}$ denote the complete $k$-graph on $p$ points. Then

$$
(2 p-(k-1)) n-1 \leqslant r\left(n K_{p}^{(k)}\right) \leqslant(2 p-(k-1)) n+C .
$$

We suppress the proof which involves only a calculation of $D$.

5. Decomposition of $K_{n}$ into monochromatic $K_{k}$. The following question was first raised for the case $k=3$ by J. W. Moon [5]: What is the minimal integer $f(n, k), k<n$, such that given a two-coloring of $K_{n}$ it is possible to find vertex-disjoint monochromatic $K_{k}$ with $\leqslant f(n, k)$ points left over? Note that the $K_{k}$ may be different colors. We are interested in $k$ fixed, $n$ large. Clearly $f(n, k)$ $\leqslant r(k, k)-1$, as given any coloring of $K_{n}$ we may delete monochromatic $K_{k}$ until there are $<r(k, k)$ points left.

THEOREM 6. If $k$ is given, then for sufficiently large $n$,

$$
f(n, k)=r(k, k-1)-1+\operatorname{rem}(n-r(k, k-1)+1, k),
$$

where $\operatorname{rem}(a, b)$ is the remainder when $a$ is divided by $b$.

Proof. We may color (see Figure 6 ) $K_{n}$ by

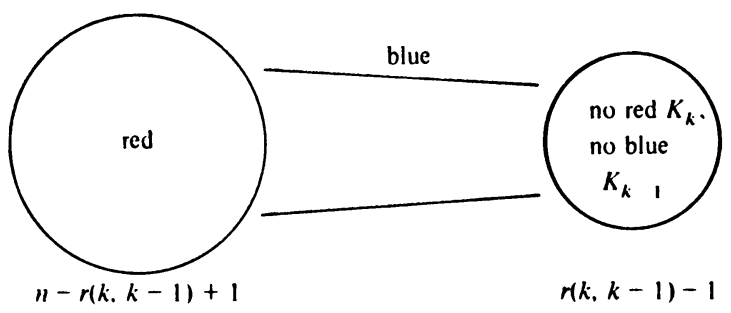

FIGURE 6

letting $|B|=r(k, k-1)-1,|A|=n-|B|, A \cap B=\varnothing$, coloring $[B]^{2}$ with no red $K_{k}$ and no blue $K_{k-1}$, coloring $A B$ blue and $[B]^{2}$ red. Then no point of $B$ can be part of a monochromatic $K_{k}$ so the $K_{k}$ must be all from $A$, leaving $|B|+$ $\operatorname{rem}(|A|, k)$ points.

Now set $u=(k-1)(r(k, k)-r(k, k-1))+(k-1)(k-2)+1$, and fix any 2-coloring of $K_{n}$, where $n \geqslant r(u, u)$. We find a monochromatic, say red, $K_{u}$ on a set $C$ of points. We decompose the remaining points into monochromatic $K_{k}$ until a set $D,|D|<r(k, k)$, is remaining with no monochromatic $K_{k}$. Assume $D$ contains a blue $K_{k-1}$ on a set $E$. For $x \in E$ let $C_{x}=\{y \in C:\{x, y\}$ is red $\}$. If any $\left|C_{x}\right| \geqslant k-1$ we take $x$ and $k-1$ points of $C_{x}$, inducing a red $K_{k}$; delete 
this and continue. If all $\left|C_{x}\right|<k-1$, then

$$
\left|C \sim \bigcup_{x \in E} C_{x}\right| \geqslant|C|-\sum_{x \in E}\left|C_{x}\right|=|C|-(k-1)(k-2)>0,
$$

so there exists $y \in C$ such that $\{y, x\}$ is blue for $x \in E$. Thus $E \cup\{y\}$ gives a blue $D_{k}$; delete this and continue.

We may continue in this manner $(|C|$ will decrease but $u$ has been chosen sufficiently large so that the above counting arguments continue to hold) until $D$ has been reduced to $D_{1},\left|D_{1}\right|<r(k, k-1)$, and $C$ to some $C_{1}$. Now $D_{1} \cup C_{1}$ are our leftovers-but we further delete red $K_{k}$ from $C_{1}$ until a set $C_{2},\left|C_{2}\right|<k$ remains. As $\left|D_{1}\right|+\left|C_{1}\right| \equiv n(\bmod k)$,

$$
\left|C_{1}\right| \equiv n-\left|D_{1}\right| \quad(\bmod k)
$$

so

$$
\left|C_{2}\right|=\operatorname{rem}\left(n-\left|D_{1}\right|, k\right)
$$

therefore

$$
\begin{aligned}
f(n, k) & \leqslant\left|D_{1}\right|+\left|C_{2}\right|=\left|D_{1}\right|+\operatorname{rem}\left(n-\left|D_{1}\right|, k\right) \\
& \leqslant r(k, k-1)-1+\operatorname{rem}(n-r(k, k-1)+1, k) . \quad \text { Q.E.D. }
\end{aligned}
$$

It would be of interest to try to extend this result to $k$-graphs.

6. Some exact values. In this section we will consider primarily some special cases of $r(n G, n H)$. To find exact values for such numbers by the methods of this paper, four steps are necessary. First, one must find a lower bound for $r(n G, n H)$ of the form $(k+l-i) n+C$. Second, one must evaluate $r\left(n_{0} G, n_{0} H\right)$ for some value of $n_{0}$ for which the lower bound is achieved. Third, one must show that $r((n+1) G,(n+1) H) \leqslant r(n G, n H)+k+l-i$ for $n \geqslant n_{0}$. Finally, one must evaluate $r(n G, n H)$ for $n<n_{0}$. Of these four steps, no general methods of carrying out the second and fourth are known, and each problem must be met on an ad hoc basis. About the first and third, however, it is possible to say something general of substance, albeit not as much as one would like.

Lemma 2 gives a very useful lower bound on $r(n G, n H)$, one which probably determines its ultimate value in a great many cases. But as Theorem 2 shows, it is sometimes possible to do better. The lower bound of Theorem 2 can be generalized, although somewhat clumsily. We first note that Ramsey numbers still make sense if one or both arguments are replaced by some class of graphs; such a generalization is indicated in [1].

Lemma 3. Let $G$ and $H$ be graphs with $p(G)=k$. Let $H$ be the class of 
maximal graphs formed by $n H$ by removing at most $m k-1$ independent points in all possible ways. Then $r(m G, n H) \geqslant m k+r(G, H)-1$.

Proof. Obvious.

Although this result appears awkward to apply, this is not always the case. For instance, in Theorem 2, in which $m=n$ and $G=H=K_{3}, H$ consists of the single graph $n K_{2}$, and $r(G, H)$ is easily seen to be $2 n+1$, yielding $r\left(n K_{3}\right) \geqslant 5 n$ as desired.

To prove that $r((m+1) G,(n+1) H) \leqslant r(m G, n H)+k+l-i$ for appropriate $m$ and $n$, it is sufficient to prove that a two-colored complete graph on that many points has either a red $(m+1) G$, a blue $(n+1) H$, or a bowtie. It turns out that in many cases it can be shown that if one has a red $G$ and a disjoint blue $H$ one must have a bowtie between them. In addition it is easy to see that any graph on that many points must have a red $(m+1) G$, a blue $(n+1) H$, or a red $G$ and a blue $H$ disjoint from each other. This leads us immediately to the following result.

Lemma 4. Let $p(G)=k, p(H)=l, i=\min \left(\beta_{0}(G), \beta_{0}(H)\right)$, and suppose that any two-colored graph containing a mutually disjoint red $G$ and blue $H$ contains a bowtie. Then, if $m \geqslant 1, n \geqslant 1$,

$$
r((m+1) G,(n+1) H) \leqslant r(m G, n H)+k+l-i .
$$

Unfortunately the conditions of Lemma 4 are not always met, for instance for $G=H=K_{4}$; nevertheless they often are, as will be seen. We will now begin the study of specific cases by completing the proof of Theorem 2, and generalizing it.

THEOREM 7. Let $m \geqslant n \geqslant 1, m \geqslant 2$. Then $r\left(m K_{3}, n K_{3}\right)=3 m+2 n$.

Proof. We first apply Lemma 3. H consists of the single graph $n K_{2}$, and it is easy to see that $r\left(K_{3}, n K_{2}\right) \geqslant 2 n+1$, so $r\left(m K_{3}, n K_{3}\right) \geqslant 3 m+2 n$.

The hypotheses of Lemma 4 have been shown, by the proof of Theorem 2, to be satisfied. Thus $r\left((m+1) K_{3},(n+1) K_{3}\right) \leqslant r\left(m K_{3}, n K_{3}\right)+5$. Hence the desired result will follow from the initial conditions $r\left(2 K_{3}\right) \leqslant 10, r\left(m K_{3}, K_{3}\right) \leqslant$ $3 m+2$. We first show $r\left(2 K_{3}\right) \leqslant 10$, which also completes the proof of Theorem 2.

Fix a two-coloring of $K_{10}$ and assume there is no monochromatic $2 K_{3}$. We easily see that if there were no monochromatic $2 K_{3}$, there would exist a bowtie, which we now fix. The five points not in the bowtie must not contain a monochromatic $K_{3}$, so our $K_{10}$ must be as in Figure 7 .

By symmetry we may assume three of the edges $0 i, 5 \leqslant i \leqslant 9$, are blue. If $0 i_{1}$, 


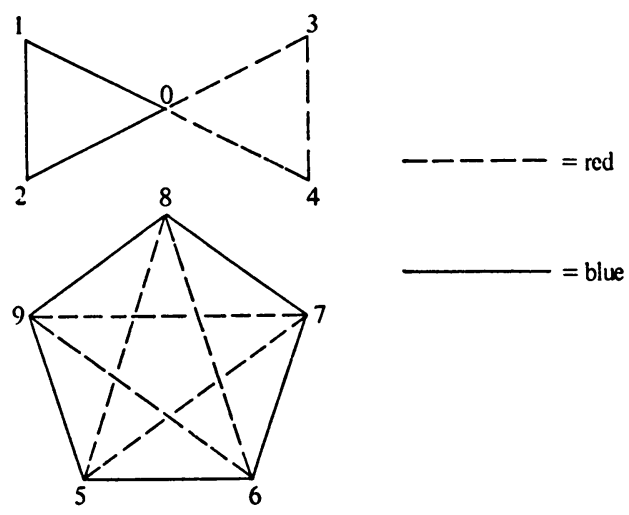

FIGURE 7

$0 i_{2}, 0 i_{3}$ are blue, two of $i_{1}, i_{2}, i_{3}$ are adjacent in the blue graph so, by symmetry, we assume 05,06 blue. Now 56034 is a bowtie so 12789 must be two-colored without a monochromatic $K_{3}$, so the blue lines must form a pentagon. As 12, 78,89 are already blue either 19,27 or 17,29 are blue. The cases are symmetric, so we assume the latter, with the remaining edges of 12789 red. Now, 19, 96 red implies 61 blue (otherwise 169, 034 red) so 61034 is a bowtie, hence 25789 is a blue pentagon; but $92,95,98$ are already blue, a contradiction.

We next show that $r\left(2 K_{3}, K_{3}\right) \leqslant 8$. In [5] it was shown that if $K_{8}$ is twocolored, it contains two disjoint monochromatic triangles. If both are red, we are done; if one is blue, we are also done. Therefore $r\left(2 K_{3}, K_{3}\right) \leqslant 8$; but then successive applications of the first part of Lemma 1 yield $r\left(m K_{3}, K_{3}\right) \leqslant 3 m+2$ for $m \geqslant 3$ as desired. Q.E.D.

THEOREM 8.

$$
\begin{aligned}
r\left(K_{1,3}\right) & =6, \\
r\left(m K_{1,3}, n K_{1,3}\right) & =4 m+n-1, \quad m \geqslant n, m \geqslant 2 .
\end{aligned}
$$

Proof. The first part is easy; see [6]. In [7] it is established that $r\left(2 K_{1,3}\right)=9$, and Lemma 2 shows that $r\left(m K_{1,3}, n K_{1,3}\right) \geqslant 4 m+n-1$. Also, an easy calculation, which we omit, shows that $r\left(2 K_{1,3}, K_{1,3}\right)=8$. Successive applications of the first part of Lemma 1 establish that $r\left(m K_{1,3}, K_{1,3}\right) \leqslant 4 m$. Another easy calculation shows that the conditions of Lemma 4 are satisfied, so that $r\left((m+1) K_{1,3},(n+1) K_{1,3}\right) \leqslant r\left(m K_{1,3}, n K_{1,3}\right)+5$, and the theorem follows by induction. Q.E.D.

THEOREM 9. If $p(G)=k$, then $r\left(n G, n K_{2}\right)=(k+1) n-1$.

Proof. By Lemma $2, r\left(n G, n K_{2}\right) \geqslant(k+1) n-1$. To prove the other 
half of the result it suffices to let $G=K_{k}$. Certainly $r\left(K_{k}, K_{2}\right)=k$; moreover, the conditions of Lemma 4 are clearly satisfied, so the result follows immediately. Q.E.D.

Let $P_{3}$ denote a path on three points (not edges). Chvátal and Harary [8] have shown that if $p(G)=k$, then $r\left(G, P_{3}\right)=k$ if $\bar{G}$ has a 1 -factor, and $r\left(G, P_{3}\right)=$ $2 k-2 \beta_{1}(\bar{G})-1$ otherwise, where $\beta_{1}(\bar{G})$ is the number of lines in a maximal independent set in $\bar{G}$. In our final theorem, we extend this result.

THEOREM 10. If $p(G)=k$, then, provided $n \geqslant 2$,

$$
r\left(n G, n P_{3}\right)= \begin{cases}(k+2) n-1 & \text { if } G=K_{k}, \\ (k+1) n-1 & \text { if } G \neq K_{k} .\end{cases}
$$

Proof. The lower bound follows easily from Lemma 2. For the upper bound, first assume $G=K_{k}$. Fix a two-coloring of $K_{2 k+3}$. By the result of Chvátal and Harary quoted above, $r\left(2 K_{k}, P_{3}\right)=2 k$, so we can assume we have a blue $P_{3}$. Remove these three points, leaving a two-coloring of $K_{2 k}$. But by the same result there must now exist a red $2 K_{k}$ or another blue $P_{3}$. Thus $r\left(2 K_{k}, 2 P_{3}\right)$ $=2 k$. It is easy to see that the conditions of Lemma 4 are satisfied; thus we are immediately led to the result $r\left(n K_{k}, n P_{3}\right)=(k+2) n-1$.

We now must show $r\left(n G, n P_{3}\right) \leqslant(k+1) n-1$ if $G \neq K_{k}$; we will omit many details. We may assume that $G=K_{k}-x$; that is, the graph formed by removing one edge from $K_{k}$. Again, Lemma 4 is applicable, leaving only the problem of establishing that $r\left(2\left(K_{k}-x\right), 2 P_{3}\right)=2 k+1$. Fix a two-coloring on $K_{2 k+1}$ and assume that there is neither a red $2\left(K_{k}-x\right)$ nor a blue $2 P_{3}$. Consider the largest component $C$ of the blue subgraph. All other blue components have one or two points. Let $v$ be a point of maximal (blue) degree in $C$. The degree of $v$ must be at least 3. Now consider the graph formed by removing $v$ from $C$. This graph must contain a $P_{\mathbf{3}}$. At most one blue line emanating from $v$ can go to a point not in that $P_{\mathbf{3}}$. This leads to two cases; each one can easily be seen to lead to a blue graph whose complement contains a red $2\left(K_{k}-x\right)$, a contradicition. Q.E.D.

An exact result of particular interest to obtain would be the value of $r\left(n K_{4}\right)$, at least for large $n$. It is easy to see, for instance by Lemma 3, that $r\left(m K_{k}, n K_{l}\right) \geqslant k m+l n-\min (m, n)+r(k-1, l-1)-2$ in general. It is conjectured that for large $m$ and $n$ equality is achieved; so perhaps $r\left(n K_{4}\right)=7 n+4$ for large $n$.

The results of this section are of interest in themselves, but they are also significant in that they indicate a deficiency in the rest of the paper. The bounds on $C$ and $n_{0}$ that come out of the proof of Theorems 1 and 4 are very large, being essentially double exponentials, and the $n_{1}$ of Theorem 3 has no known 
bound at all. These bounds essentially come from the proof of the existence of a bowtie. In this section we have seen that often it requires relatively few points to force a bowtie. This is also true in [9], where similar ideas occur. It seems quite possible that more reasonable general bounds can be found for the constants in Theorems 1 and 4, or even for Theorem 3.

\section{REFERENCES}

1. V. Chvátal and F. Harary, Generalized Ramsey theory for graphs, Bull. Amer. Math. Soc. 78 (1972), 423-426. MR 45 \#110.

2. S. A. Burr, Generalized Ramsey theory for graphs a survey, Graphs and Combinatorics, R. Bari and F. Harary, eds., Lecture Notes in Math., vol. 406, Springer-Verlag, Berlin and New York, 1974, pp. 52-75.

3. F. Harary, Graph theory, Addison-Wesley, Reading, Mass., 1969. MR 41 \#1566.

4. E. J. Cockayne, An application of Ramsey's theorem, Canad. Math. Bull. 13 (1970), 145-146. MR 41 \#6721.

5. J. W. Moon, Disjoint triangles in chromatic graphs, Math. Mag. 39 (1966), 259261. MR $35 \# 4123$.

6. V. Chvátal and F. Harary, Generalized Ramsey numbers for graphs. II: Small diagonal numbers, Proc. Amer. Math. Soc. 32 (1972), 389-394.

7. S. A. Burr, Diagonal Ramsey numbers for small graphs (to appear).

8. V. Chvátal and F. Harary, Genveralized Ramsey theory for graphs. III: Small offdiagonal numbers, Pacific J. Math. 41 (1972), 335-345. MR 47 \#3248.

9. S. A. Burr and J. A. Roberts, On Ramsey numbers for Linear forests, Discrete Math. 8 (1974), 245-250.

BELL TELEPHONE LABORATORIES, 175 PARK AVENUE, MADISON, NEW JERSEY 07940 of P. Erdös)

HUNGARIAN ACADEMY OF SCIENCES, BUDAPEST, HUNGARY (Current address

DEPARTMENT OF MATHEMATICS, MASSACHUSETTS INSTITUTE OF TECHNOLOGY, CAMBRIDGE, MASSACHUSETTS 02139

Current address (S. A. Burr): A.T.\&T. Long Line, 110 Belmont Drive, Somerset, New Jersey 08873

Current address (J. H. Spencer): Department of Mathematics, SUNY at Stony Brook, Stony Brook, New York 11790 